\title{
Fast Online Trigger using FPGA-based Event Classification for the COMET Phase-I
}

\author{
Yu Nakazawa*, Tai Thanh Chau \\ Department of Physics, Osaka University \\ E-mail: y-nakazawa@kuno-g.phys.sci.osaka-u.ac.jp
}

\section{Yuki Fujii}

School of Physics and Astronomy, Monash University

\section{Ewen Gillies}

Department of Physics, Imperial College London

Masahiro Ikeno, Satoshi Mihara, Masayoshi Shoji, Tomohisa Uchida, Kazuki Ueno

Institute of Particle and Nuclear Studies, High Energy Accelerator Research Organization

\section{MyeongJae Lee}

Center for Axion and Precision Physics Research, Institute for Basic Science

\begin{abstract}
The COMET Phase-I experiment searches for a neutrinoless muon-to-electron conversion which has never been observed yet. The world's highest intensity muon beam is applied, and it leads to an unacceptable trigger rate of $\mathscr{O}\left(10^{6}\right) \mathrm{Hz}$. For stable data collection, the trigger rate must be reduced to $\mathscr{O}\left(10^{3}\right) \mathrm{Hz}$. This requirement is met using online event classification in the detector system which holds $99 \%$ of signal events. This classification is performed by an FPGA-based trigger system, and its processing time is set to less than $5 \mu$ s by a buffer size of the detector readout electronics. A prototype board for the trigger system was developed, and communication systems for related electronics devices were also constructed. From test results, the total processing time is estimated to be $2.8 \mu \mathrm{s}$, which meets the requirement. We have also developed an online self-trigger system for cosmic-rays and confirmed the feasibility of this hardware logic. The trigger electronics were installed in a setup for cosmic-ray measurement, and the data acquisition was successfully done using the self-trigger system.
\end{abstract}

European Physical Society Conference on High Energy Physics - EPS-HEP2019 -

10-17 July, 2019

Ghent, Belgium

${ }^{*}$ Speaker. 


\section{Introduction}

The COMET Phase-I experiment searches for a neutrinoless muon-to-electron conversion at a target sensitivity of $3 \times 10^{-15}$, which has never been observed $[1,2]$. The event signature is the emission of a mono-energetic electron of $105 \mathrm{MeV}$ from a muonic atom of aluminium. In order to reduce irrelevant particles that may generate background events in a measurement window, a pulsed muon beam is selected, and the window is delayed from the pulse timing. The signal electrons are detected by a Cylindrical Detector (CyDet) system consisting of a Cylindrical Drift Chamber (CDC) and a CyDet Trigger Hodoscope (CTH) in a $1 \mathrm{~T}$ solenoidal magnetic field. The CDC has 4,986 sense wires and 14,562 field wires in total, and all the wires are strung with an alternated all stereo layer configuration to measure particle momenta in the longitudinal direction. The readout electronics for the central drift chamber of the Belle II detector (RECBE) [3] are used as the COMET CDC readout electronics with digital-logic modifications. The CTH consists of 48 sets of Cherenkov and scintillation counters and located at both the upstream and downstream ends of the CDC. These counters detect the electrons using a simple 2-fold hit coincidence. Figure 1 shows a schematic layout of the COMET Phase-I setup.

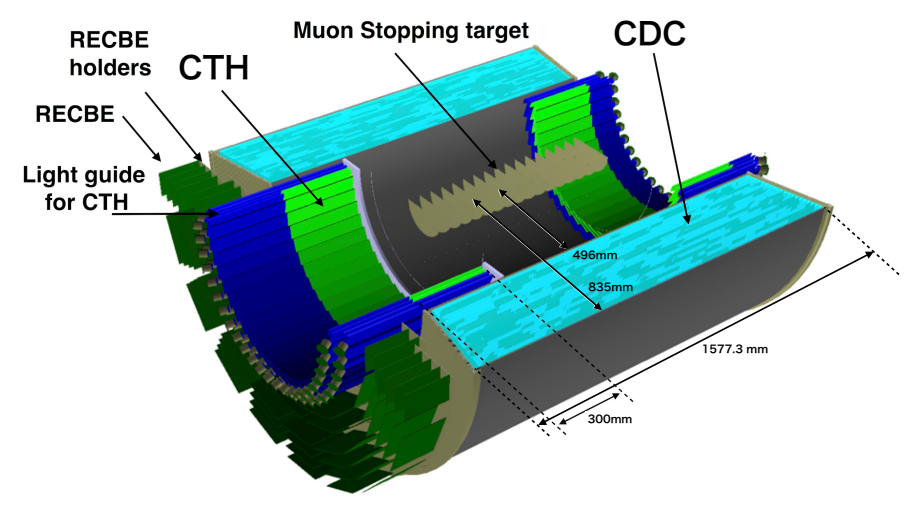

Figure 1: A schematic layout of the COMET Phase-I setup [1].

The highly intense muon beam is used to achieve our sensitivity goal. It leads to an unacceptable trigger rate of a few $\mathrm{MHz}$, while a system of data acquisition requires the trigger rate of less than $24 \mathrm{kHz}$. Triggered events are mainly generated by electrons from the reaction between CTH and gamma-rays emitted from the target. When a 4-fold hit coincidence ( 2 sets of Cherenkov and scintillator rings) is adopted, an average of the trigger rate is expected to be $30 \mathrm{kHz}$ from a simulation study. This rate varies greatly depending on simulation conditions and measurement time window. It is required to develop a trigger system that makes the trigger rate small enough for the stable data acquisition, considering these trigger-rate uncertainties. The total processing time of the trigger system has to be less than $5 \mu$ s due to buffer sizes of RECBE. A fast online trigger system that meets these requirements is being developed in COMET Phase-I [4, 5]. This paper shows the expected system performance during the physics measurement and the test results with a CDC setup for cosmic-ray measurements.

\section{Fast online trigger scheme}

The fast online trigger system, called the COMET trigger (COTTRI) system, performs event 
classification using Gradient Boosted Decision Trees (GBDT) and Field Programmable Gate Arrays (FPGAs). For the event classification, there are two phases; local and global classification phases. In the local phase, the COTTRI system evaluates CDC hits using the GBDT whose output indicates the hit quality. Background particles, mainly protons and low energy electrons, tend to make larger energy deposition than the signal electrons in the CDC. Moreover, because of helical tracks of the signal electrons from the $\mu-e$ conversion, the track projections have fewer hits on the outermost layer of the CDC. Therefore, energy deposition and radial positions of evaluated hits are selected as the GBDT input features. The energy deposition on adjacent wires is also applied to the local classification. Since the helical tracks make more continuous hits along tangential directions, both the left and right neighbours in the same layer of the evaluated wires are used. Due to the data transfer rate of RECBE described in the next section, the data size of the energy deposition is set to 2 bit for each CDC wire. On FPGAs in the trigger electronics, Look-Up Tables (LUTs) indicate the relationship between the input features and the GBDT outputs and convert from input to output within a clock cycle. The LUT has 6 inputs consisting of 2-bit local information and 4-bit (2×2-bit) neighboring information. When the GBDT output size is 6 bit, 6 LUTs inside the FPGA are used for each CDC wire. The LUTs are changed for each hit radial position, then 18 different LUT sets are needed for the 18 CDC layers. The features are shown in Figure 2.

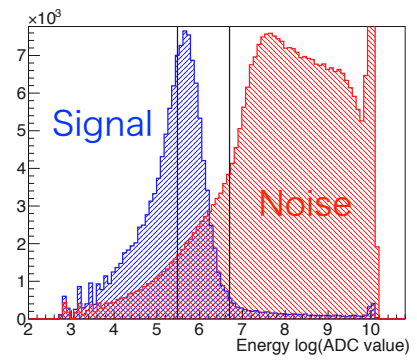

(a)

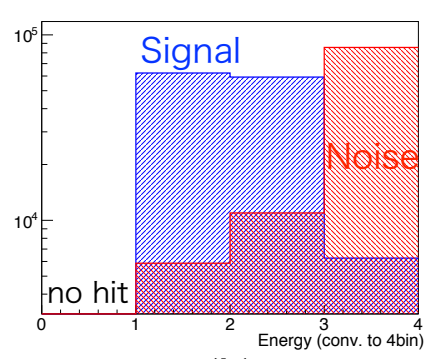

(b)

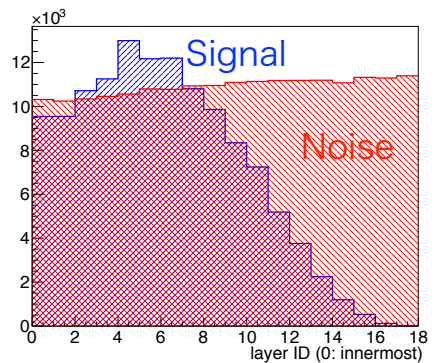

(c)

Figure 2: Input features for the GBDT in the local classification phase. (a) Uncompressed and (b) Compressed distributions of energy deposition in a CDC cell, and (c) Distribution of hit radial positions in the CDC. Blue and red histograms show the distributions of the signal and noise hits, respectively. 2 black lines in (a) show 2 threshold values to compress the information of the energy deposition.

The GBDT outputs from all the CDC wires are evaluated with relative trigger timing from the $\mathrm{CTH}$ in the global classification phase. A hit whose GBDT output exceeds a threshold is defined as a signal-like hit. A trigger signal is generated when the sum of all signal-like hit GBDT outputs exceeds another threshold. This threshold for the total GBDT output is selected at the point where the acceptance of signal events is $99 \%$. The simulation study shows that the classification can reject about $93 \%$ of background events. This corresponds to the trigger rate of $1.9 \mathrm{kHz}$ in conjunction with the information of CTH. Figure 3 shows the results in the local and global classification phases.

\section{Trigger system in COMET Phase-I}

Figure 4 shows a conceptual design of the COTTRI system. First, RECBEs provide 10-bit pulse-shape information for each CDC wire at $30 \mathrm{MSPS}$, which corresponds to the energy deposition. 1 RECBE board handles up to 48 CDC wires, and 104 RECBE boards are used in to- 


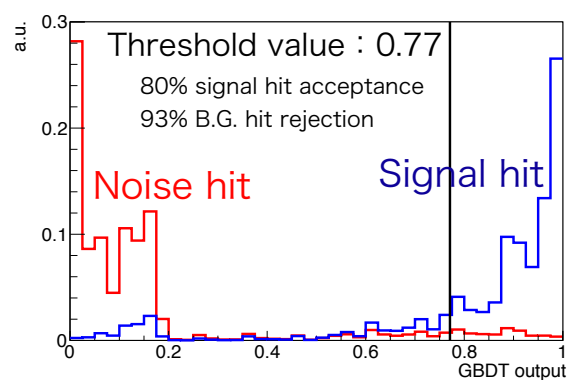

(a)

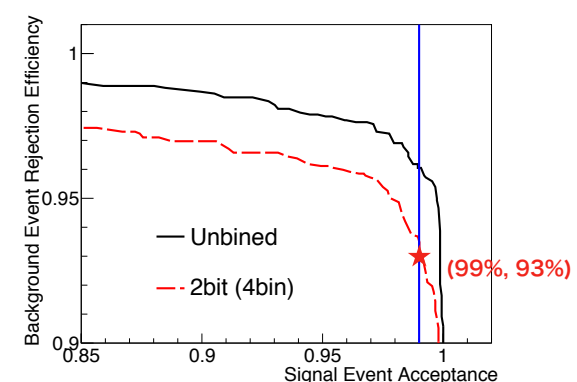

(b)

Figure 3: Results of the simulation study. (a) Distribution of GBDT outputs. The blue and red histograms show the distributions of the signal and noise hits, respectively. (b) A receiver operating characteristic (ROC) curve of the event classification. A black line shows the ROC curve calculated by using the uncompressed distribution of the energy deposition, and red one shows the ROC curve calculated by using the compressed distribution of the energy deposition.

tal. 3 samples of the pulse-shape data are converted to 2-bit energy data by an FPGA (Virtex-5, xc5vlx155t-1ffg1738, Xilinx Inc. [6]). RECBE sends it to the COTTRI Front-End (FE) board every $100 \mathrm{~ns}$ with a data transfer rate of $2.4 \mathrm{Gbps} / \mathrm{lane}$ which is decided by the Virtex-5 specification and the clock frequency of $120 \mathrm{MHz}$. In the local classification performed by the COTTRI FE board, the received data is converted to the GBDT output by the LUT, and these outputs are summed for each RECBE. The COTTRI FEs send the total value to the COTTRI Merger-Board (MB). The COTTRI MB performs the global classification without the CTH timing information and makes the CDC trigger decisions. The CDC trigger signal is sent to FC7 developed in CERN [7]. Using both the CDC trigger information and the $\mathrm{CTH}$ trigger information, FC7 makes the final trigger decisions and distributes the trigger signals to all RECBEs through sets of FCT (Fast Control and Timing) boards. In the trigger system, 1 COTTRI FE board is connected to 10 RECBE boards. 1 COTTRI MB is connected to 10 COTTRI FE boards and covers 100 RECBE boards. The 4 RECBE boards connected to wires in the outermost CDC layers are disconnected from the COTTRI system because they make negligible contributions to the event classification as shown in Figure 2 (c).

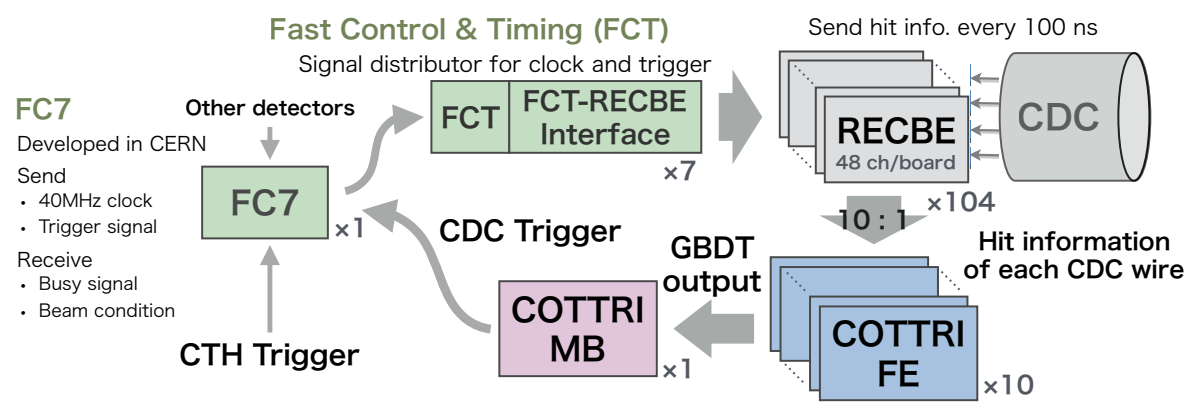

Figure 4: A conceptual design of the COTTRI system.

Last summer, a prototype of the COTTRI MB was developed as shown in Figure 5. This board has 1 FPGA (Kintex-7, xc7k355tffg901, Xilinx Inc.), 10 DisplayPort connectors, 2 SFP+ ports, and a clock multiplier/jitter attenuator (Si5326 [8]). Si5326 generates a low jitter clock from the $40 \mathrm{MHz}$ clock distributed by FC7 to completely synchronize the data transfer between the board 
and other devices in the trigger system. The required functions of COTTRI FE and MB are similar, therefore, this COTTRI MB prototype can be used for the performance study of COTTRI FE. When used as COTTRI FE, it is necessary to change the classification part of the firmware for the local classification.

\section{Performance tests of the COMET trigger system}

The total processing time was measured by connecting 1 RECBE, 1 COTTRI MB prototype, 1 FC7, and a set of FCT boards. This setup corresponds to the almost final configuration but without the COTTRI FE stage. In this test, the COTTRI MB prototype performs both the COTTRI FE and MB functions, and the Aurora 8B/10B protocol [9] using $120 \mathrm{MHz}$ GT reference clock, $2.4 \mathrm{Gbps} / \mathrm{lane}$, and 2 lane/cable was used for the communication between RECBEs and the COTTRI MB prototype. Figure 6 shows the timing of the test pulse from the function generator to RECBE, the trigger from the COTTRI MB prototype, and the trigger data from FC7. As the test result, the processing time without COTTRI FE was measured to be $1.2 \mu \mathrm{s}$. In addition to this, the total processing time of the trigger system needs to be estimated, taking into account the drift time in the CDC cell, trigger-receiving time, and data-transfer time between COTTRI FE and MB. The typical drift time is about $400 \mathrm{~ns}$. When using the $40 \mathrm{MHz}$ clock from FC7, it takes $800 \mathrm{~ns}$ to receive the trigger data which is a 32-bit trigger number. Then, considering the COTTRI MB process similar to the COTTRI FE process, the processing time of the full trigger system is expected to be approximately $2.8 \mu \mathrm{s}$ while the required time is less than $5 \mu \mathrm{s}$.

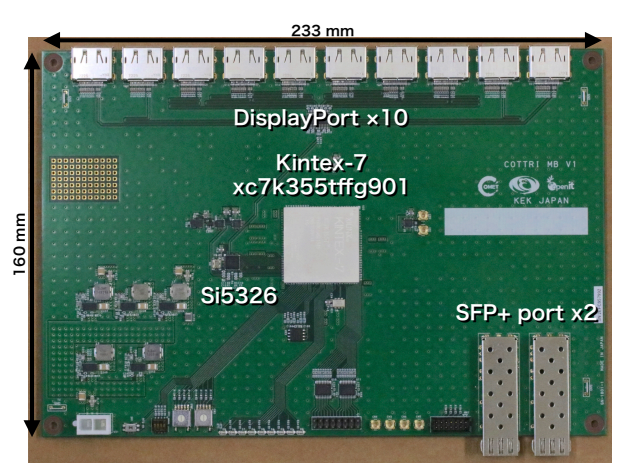

Figure 5: A photo of the COTTRI MB prototype board.

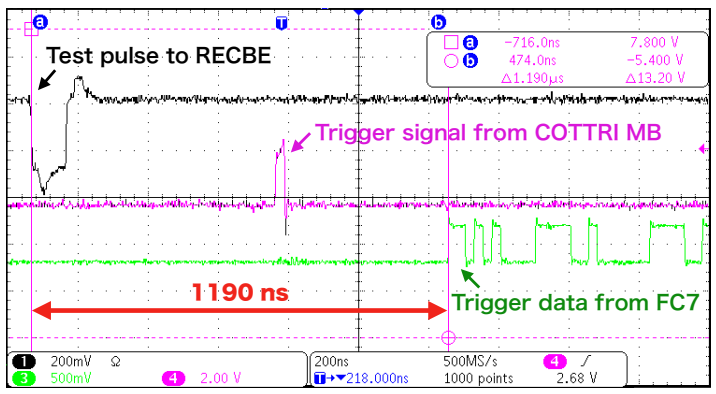

Figure 6: A result of processing-time measurement. A black line shows the timing of a test pulse on RECBE, a magenta line shows the timing of a trigger signal from the COTTRI MB prototype, and the green line shows the timing of trigger data from FC7.

In order to verify that the system can apply the trigger, the system was installed in the CDC setup for cosmic-ray measurements. Implementing a pseudo-algorithm of the trigger system on the COTTRI MB prototype, taking cosmic-ray data was successfully done by using 1 COTTRI MB, 1 FC7, 1 set of FCT, and 6 RECBEs. The pseudo-algorithm has LUTs that counts the number of CDC hits instead of the optimized GBDT LUTs. Except for this LUT difference, the hardware logic of the trigger algorithm is the same as the one for the physics measurement. Scintillation counters were used to measure the timing of cosmic-rays passing through the CDC. The hit-timing distribution from the COTTRI MB data has the same shape as the drift-time distribution from the CDC cosmic-ray data, as shown in Figure 7 (a). Tracking the cosmic-ray events was succeeded 
using the data from the $\mathrm{CDC}$ and the scintillation counters acquired using the fast online trigger system. A reconstructed event is shown in Figure 7 (b). The online trigger efficiency is calculated to be $99.3 \%$ which includes the inefficiency due to the uncovered CDC readout region with respect to the acceptance of the scintillation counters.

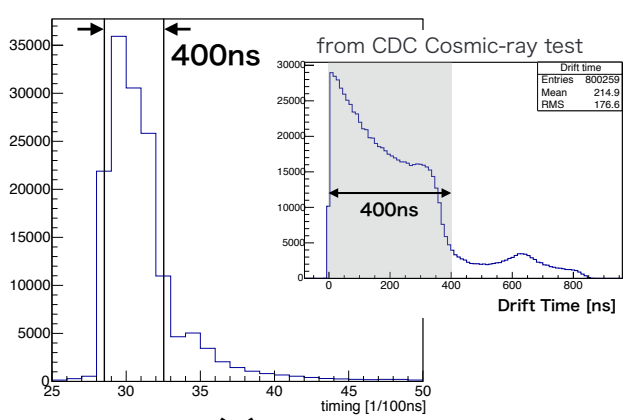

(a)

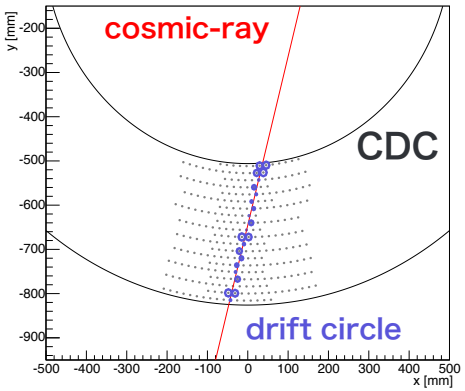

(b)

Figure 7: Results of cosmic-ray measurement. (a) Hit-timing distribution constructed by using the COTTRI MB data (left) and drift-time distribution constructed by using the RECBE data in the other CDC cosmic-ray test. (b) A display of a reconstructed comic-ray event. A red line shows the cosmic-ray track, blue circles show drift circles, and gray plots shows readout wires in this measurement.

\section{Conclusion}

The COMET Phase-I experiment searches for the neutrinoless $\mu-e$ conversion. The fast online trigger system is required for the stable data acquisition. From the simulation study, it is found that the classification rejects over $90 \%$ of background events with $99 \%$ signal acceptance. This corresponds to the trigger rate of $1.9 \mathrm{kHz}$ that meets the requirement, considering the use of the CTH information. The prototype of COTTRI MB was developed for this classification. The total processing time for the full trigger system is estimated to be $2.8 \mu \mathrm{s}$. Furthermore, we successfully took cosmic-ray data using the trigger system installed in the CDC setup. The trigger efficiency of the system is confirmed to be over $99 \%$. We concluded that the fast online trigger system using the GBDT-based event classification meets the requirements.

\section{Acknowledgement}

This work was supported by JSPS KAKENHI Grant Numbers JP17H04841, JP17H06135, JP18H05231 and JP18J10962.

\section{References}

[1] G. Adamov, et al., "COMET Phase-I Technical Design Report", arXiv:1812.09018.

[2] Y. Kuno, Y. Okada, "Muon decay and physics beyond the standard model", Rev. Mod. Phys., 73 (2001) 151. doi:10.1103/RevModPhys.73.151

[3] T. Uchida, et al., "Readout electronics for the central drift chamber of the Belle II detector", 2011 IEEE Nucl. Sci. Symp. Conference Record, NP2.S-33. doi:10.1109/NSSMIC.2011.6154084 
[4] E.L. Gillies, "COMET Phase-I Track Reconstruction using Machine Learning and Computer Vision", PhD thesis in Physics, Imperial College London, October 2018 (not published yet).

[5] Y. Fujii, et al., "Development of the Fast Front-end Trigger System for COMET Phase-I", 2018 IEEE NSS/MIC Proceedings, Sydney, 2018, doi:10.1109/NSSMIC.2018.8824619

[6] Xilinx Inc., https://www.xilinx.com.

[7] "The FC7 project", https://espace.cern.ch/project-FC7/SitePages/Home.aspx.

[8] Silicon Laboratories, "ANY-FREQUENCY PRECISION CLOCK MULTIPLIER/JITTER ATTENUATOR",201-

[9] Xilinx Inc., "Aurora 8B/10B",

https://www.xilinx.com/products/intellectual-property/aurora8b10b.html. 